\title{
Designing Smart Home (IOT) Using Cisco Packet Tracer 7.2 Simulator
}

\author{
Tanishka ${ }^{1}$ | Prof. Shikha Gupta ${ }^{2}$
}

${ }^{1}$ B.Tech Scholar, Department of IT, Maharaja Agrasen Institute of Technology, Delhi, India

${ }^{2}$ Assistant Professor, Department of IT, Maharaja Agrasen Institute of Technology, Delhi, India

\section{To Cite this Article}

Tanishka and Prof. Shikha Gupta, "Designing Smart Home (IOT) Using Cisco Packet Tracer 7.2 Simulator", International Journal for Modern Trends in Science and Technology, 6(12): 208-218, 2020.

\section{Article Info}

Received on 10-November-2020, Revised on 02-December-2020, Accepted on 06-December-2020, Published on 10-December-2020.

\section{ABSTRACT}

The internet of things, or IoT, is a system of interrelated computing devices, mechanical and digital machines, objects, animals or people that are provided with unique identifiers (UIDs) and the ability to transfer data over a network without requiring human-to-human or human-to-computer interaction. Internet of Things (IoT) is rapidly gaining momentum in the scenario of telecommunications. Conventional networks allow for interactivity and data exchange, but these networks have not been designed for the new features and functions of IoT devices. In this paper, an algorithm is proposed to share common recourse among Things, that is, between different types of smart appliances. . Purpose is to analyze deeper the cases separating the network and IoT layout, giving a deeper explanation of the purpose of the simulations, presenting all the information needed to utilize the exercises but also giving suggestion how to expand the exercises further.

This implementation can be implemented effectively using package tracking software that includes IoT functions to control and simulate a smart home. IoT technology can be applied to many real life issues, such as: homework, treatment, campus, office, etc.

KEYWORDS: Internet of Things, Home Gateway, resources sharing, IoT gateway, collaborative groups, architecture with centralized management, Cisco Packet Tracer.

\section{INTRODUCTION}

The Internet of Things (IoTs) is a novel paradigm of integration of several technologies that is rapidly gaining momentum in the scenario of telecommunications, especially in wireless network [1]. In traditional communications systems, usually within a house or an office several personal computers and smart devices are seamlessly connected together through a wireless router. In this network, resources can be shared; the most common example is a printer and access to the Internet.

Therefore, the integrated intermediary network devices, such as switches and routers, are used to interconnect the network computers in order to exchange data. These devices

Devices and objects with built in sensors are connected to an Internet of Things platform, which integrates data from the different devices and applies analytics to share the most valuable information with applications built to address specific needs. These powerful IoT platforms can pinpoint exactly what information is useful and what can safely be ignored. This information can be used to detect patterns, make recommendations, and detect possible problems before they occur. 
For example, if I own a car manufacturing business, I might want to know which optional components (leather seats or alloy wheels, for example) are the most popular. Using Internet of Things technology, This reprogramming can be done by the user directly in a configuration panel or by software from a mobile application.

The issue currently with an IoT gateway, is that not smartly self-managed or allowed to take efficient control of the exchange of resources between heterogeneous things of an IoT network.

Although, there is a variety of research about IoT Gateway for smart devices. A novel Machine-to-Machine (M2M) service is introduced in [3]. They proposed self-configurable gateway and configuration of smart things over the wireless networks. As well, an IoT gateway is designed and implemented through creating a unified connection to the technological layer aided by IoT in [4]. This proposed gateway allows management of devices and identification of new device by creating virtual representations of the physical devices. An auto configuration solution based on interpretable configuration is presented in [5], which focused on some algorithms for computing the IoT gateway configurations. Five algorithms are contributed, while a thorough evaluation reveals which of the algorithms should be used in different operation scenarios in order to achieve high fulfillment of the operator's target. Therefore, a case study is provided, to better known how to design and implement IoT-gateway for home environment, which is based on suitable self-configuration and scalability.

In this paper, we propose a new algorithm for allocating and sharing the common resources among the heterogeneous devices in the scenarios of IoT such as smart cities, smart grids, industrial automation, smart driving, elderly assistance, or home automation, and others. The proposal is based on an IoT network with centralized management architecture, controlled by an Artificial Intelligence (AI). The AI controller is an algorithm based on machine learning techniques, collecting information on the network through an information protocol. Every smart thing that connects to the IoT network deployed message through Protocol of Discovery of Functions and Services (DFSP). The information of the available and common resources is shared among the things in the integrated network.

The rest of the paper is structured as follows. Related work with IoT Gateways resources sharing in smart cities, controlled by artificial intelligence, will be explained in section 2. Then, in Section 3, we state the problem and describe the resource allocation proposal method for wireless IoT network. Section 4 presents the performance of the tests and results' discussion. Finally, in Section 5, we highlight our conclusion and future work. By including different sensors in home automation, Smart Home eliminates user engagement in tracking home settings and operating home appliances.

\section{RELATED WORK}

Internet of things is growing very fast and IoT gateway becomes an important part in its structure. IoT gateway allows supporting a variety of communication protocols and data exchange between various IoT-nodes, The goal of IoT gateway is to bridge various sensing domain networks with public communication networks or Internet, settle with the heterogeneity between these various networks, strengthen the management of both IoT gateway itself and terminal nodes [3]. Generally, Garcia-García et al. [1] presented an overview of the premise of the emerging of the Internet of Things (IoT) according to enabling technologies, protocols, and applications. They provided a good foundation to gain an insight into the IoT technologies and protocols to understand the overall architecture and role of the different components and protocols that constitute the IoT.

Zhong et al. [7] outlined the disadvantages of the practical application with three-layer architecture of IoT. In order to better interpret the meaning and features of the IOT, and discusses the gateway technology which connecting the sensing network and traditional communication network, layers system architecture is explained. They designed IoT application scheme with using the IoT Gateway as a bridge in order to realize exchanges of the information and communication of different equipment in the industry services. Therefore, the sensors and devices use numerous protocols and communication methods for exchanging the data. In a traditional setup, it is difficult to connect multiple devices with different proprietary applications and monitor. Moreover, it is heavy to control multiple devices and store, analyses all the vitals parameter data together. There is no common platform available to connect the multiple sensors and to monitor the vitals simultaneously. Selvaraj and Kalambettu [8] proposed the comprehensive, scalable, plug and play smart gateway for connecting variety of health sensors and allows the 
smart devices seamless communication for transmit and control the resources. With IoT-Gateway enabled the framework seamlessly connects with cloud services and stream the data for storage, analysis and prediction. The proposed unified framework is also enable seamless connectivity between gateway and cloud platform and services.

The architecture of the heterogeneous Internet of Things (IoT) gateway and the problems of its integration with the Industrial Internet of Things (IIoT) are investigated in [9] by Viacheslav Et al. the issue of the usage of heterogeneous gateway for conversion of packages raised when various protocols are formed in IoT. Therefore, they proposed Industrial Internet of Things Conversion Format (IICF) for converting various protocols of the Industrial Internet of Things among themselves. The proposal based on the analyzed architecture of the heterogeneous IoT gateway, structure of interaction softwaresystem, structure of the model network for interoperability different protocols. Thus, Rodrigues et al. in [10] proposed a novel IoT-based mobile gateway solution for mobile health scenarios. The gateway autonomously collects

informationabouttheuser/patientlocation, heartrat e, and possible

falldetection.Moreover, itforwardsthecollectedinfor mation

toacaretakerIPA, inrealtimethatmanageasetofaction sand alarms appropriately. The algorithms used for each mobile gateway service, and the scenarios where the mobile gateway acts as a communication channel or a smart objects. Also, Athreya et al. [11] took a preliminary look at software, hardware, and network architectures involved in IoT systems. They summarize that the various network components to operate and interoperate effectively the smart devices must be able to coordinate their management capabilities.

They proposed the use of self-management and selfadaptation to cope with countless dynamics. The proposal presents an underlying framework for self-managing devices, comprising measurement based learning and adaptation to changing system context and application demands.

An IoT gateway, which provides device connectivity and protocol translation, is often expensive in case of small-scale academic projects. A method is provided to emulate the functionality of an IoT gateway using a local computer instead of a separate hardware. The proposed EmI gateway is successfully tested to provide communication between sensor nodes in heterogeneous networks such as GPRS, WiFi, Ethernet, RF, and Bluetooth and Amazon's cloud server. The EmI gateway is used in the IoT based application of sewage flow scheduling that involves sensor nodes connected in different communication networks.

Therefore, the studies are presented above on IoT gateways do not offer dynamic resource allocation information protocols for that things can use it. Therefore, it is necessary to explore proposals that allow for different ways of assigning resources and sharing dynamically among the smart devices in the networks. Especially when the current proposals already speak on IoT networks with AI intervention and M2M techniques. For this reason, we propose IoT-gateway algorithm based on artificial intelligent to allow dynamicselfmanagement of gateways when the resources needed to be assigned and shared between IoT-nodes in wireless networks.

\section{PROBLEM STATEMENT}

The technology has been growing from day to day in human life. The necessity for the development of this technology is to lead human life comfortably. One is needed to create a smart home when electronic devices are switched on and off. Smart home is a house that uses currently released IOT technology to automate different activities of home.

\section{SCOPE \& MOTIVATION}

Smart the tool chosen here for the simulation is cisco Packet tracer which has been using for many years to train the students in the field of networking by cisco industry. The versatility of this simulator is that it will offering of a variety of network components which can simulate in real time networks, devices would then need to be interconnected further it is configured to create the desired networking mechanism.The latest version of packettracer simulator supports IOE devices and controller boards such as SBC-PT and MCU-PT it also supports microcontrollers such as Arudino or Raspberry pi.The main advantages of this simulator is all theIOT devices can be programmed with java.python or blockly.

Scope of this prohect is to allow user to build,design and configure smart city and smart home by providing a different intelligent object hat uses them, and to provide realistic visualization and simulation of IOT devices. This technology 
made it possible to control our home appliances with the help of mobile applications or voice assistants. People in India are easily able to quickly adopting this Technology and can set or schedule their appliances accordingly. This will be a revolution in the future to change simple homes into smart homes to make consumers most comfortable and add convenience to their life.

\section{PROJECT OBJECTIVE}

The main objective of the functions is to improve the quality of life and convenience in the home. Other goals are greater security and more efficient use of energy thanks to connected, remote-controllable devices.

1.The project aims at designing an advanced home automation system using Cisco Packet Tracer

2. The proposed system develops a smart home system that gives the user complete control over all remotely controllable aspects of the system.

3. The objective is to presents a low cost and flexible home control and environmental monitoring system.

4. The objective of system is to control home appliances anytime from anywhere in the world and efficiently utilize power by controlling appliances properly.

5. The system provides the implementation to be available in very less cost to ruler area peoples and schools.

\section{PROPOSED SYSTEM}

The proposed system introduces the advance $A$. PROBLEM

The current data networks allow to connect "Things", but under their policies and protocols. Although protocols such as transmissioncontrol(TCP/IP)areflexibleandallowtra nsport to other protocols in the network, it is a low level protocol, which does not solve all the problems that appear in an IoT network [13]. Things must be managed differently and the resources that Things need are not the same as a conventional network. SomeIoTprotocolssuchasMQTTcanexchangemessa ges on these types of networks, as well as the Constrained Application Protocol (CoAP) and Representative State Transfer (REST) [14]. MQTT and CoAP are M2M protocol and REST uses HTTP to perform operations between client and server. However, the operation of these protocols is not designed to share, use or allocate resources on the network.
For that, is necessary a protocol or autonomous entity that allow to Things talk between them without human intervention, only machines. In this case, it has been decided to work with MQTT protocol [15], since is open source and allow modifying its operating mechanics. MQTT performs the M2M communication through a central server (MQTT

Broker)insideaGatewayordirectlythroughthecloud(

Cloud Broker). This is a publication/subscribe protocol and was initially aimed at IoT sensors networks [16], since its main target was to optimize bandwidth and minimize the hardware and the processing [1].

However, is possible that when creating an adaptive algorithm, it is can take advantage of its own messages to send over they the new messages of the DFSP protocol designed for this proposal.

Another problem is that wireless routers in home networks are not flexible and do not allow reprogramming of protocols. Commercially this type of devices are obtained, but with very limited functions. To achieve modifications in the network and that Things operate under another type of architecture, it is necessary to change the traditional router to replace it with a reprogrammable one.

In addition, Things do not have the capacity to process information because their factory functions are limited and they cannot collaborate and interact with other things in a network. It is not enough to have a means of communication to the internet, it is necessary to increase the processing capacity.

From the above, it is evident that the devices that make up a conventional data network, such as the host, the router and the cloud platform, must be reconsidered.

\section{B. PROPOSAL}

The next proposal consists of creating three control agents of the same type but in different environments. This control agent is the AI, which will be inside the smart things, in the Gateway with centralized administration and the platform in the cloud. The AI is based on the same principle and has two main functions, managing the algorithms based on Machine Learning techniques and learning the relations Machine to Machine (M2M) as the product of the joint work of a group of Things.

It is necessary to see the problem first since the perspective of the "Things", because these just 
work when its user controls it or if before it had a previous programming. For that, the things carry its own control, it is necessary to know that resource, function or service is shared between the things.

This paper addresses the study of the problem through the analysis of the exchange of DFSP protocol messages between smarts things, the gateway, and the cloud platform. In this way, it is can see how each Thing that connects announces its functions and services and shares it on the network.

On the other hand, the AI that administers the algorithm that assigns the role to each Thing decides that Thing is a resource or makes available a resource. This is an adaptive algorithm and changes according to the role that each Thing assumes within the group work.

\section{NETWORK IOT ARCHITECTURE WITH CENTRALIZED MANAGEMENT}

To understand the architecture that will use in this proposal is fundamental to explain it through of three actors and

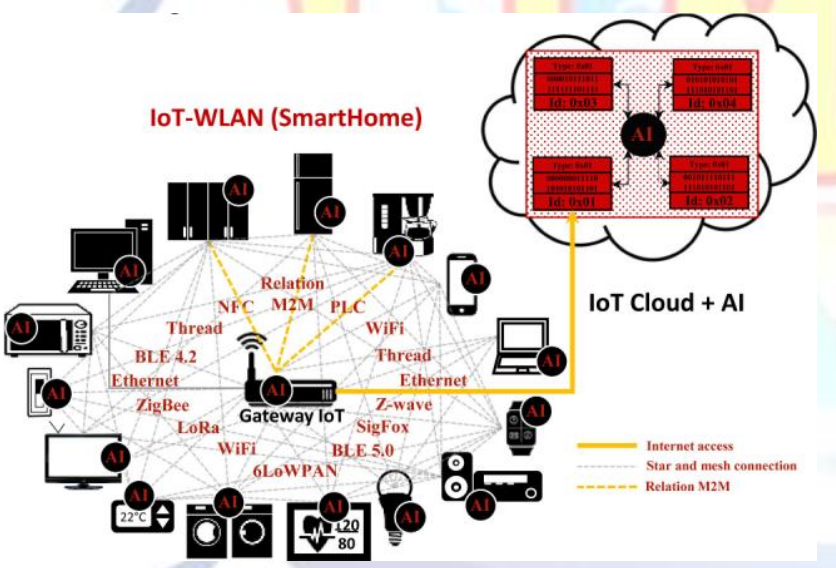

FIGURE 1. Network IoT-WLAN architecture.

its relation: Smart Things, the IoT Gateway and the IoT platform, each contain an AI.

Through the communication of the AI between these three devices, it is possible to achieve a harmony of collective learning in the entire network.

The design of this architecture is thought for a Wireless Local Area Network (WLAN), connecting Things with different interconnection technologies and IoT protocols, depending on their use, bandwidth, processing capacity, and distance [17]. The idea is to modify a network home conventional and become it in a WLAN for IoT. The Figure 1, show an example of network IoT-WLAN, with different topologies and IoT protocols.
The connection of the Gateway to the intelligent platform in the cloud is done through the Internet and constantly monitors what happens in the house. If it is realized queries to the cloud, the platform validates the type of request, classifies it and decides if it is necessary to connect to other platforms else the AI into gateway decides if the problem can be resolved on the local network through the M2M connections [18].

Smart Things + AI: The importance of things acting intelligently, not just improves its internal functions, but it also allows defining functions and services that put to disposition to other things in the network.

Things, in this case study will be appliances inside a house. These require the ability to process and communicate with different communication technologies.

In the architecture of Figure 1, it is can see how everything connects directly to other things or through the Gateway. This connection type will depend on the permissions and relations established by the Gateway.

For example, yellow line of figure 1 depicts the relation M2M established for the gateway where creating a group with something in common.

IoT Gateway + AI: This multiprotocol device allows managing and centralizing all of the information regardless of the underlying technology of interconnecting. It carries out the management in a centralized way in the network and allows all the Things in the network to connect with each other, initially passing through it. In this way, the M2M relation

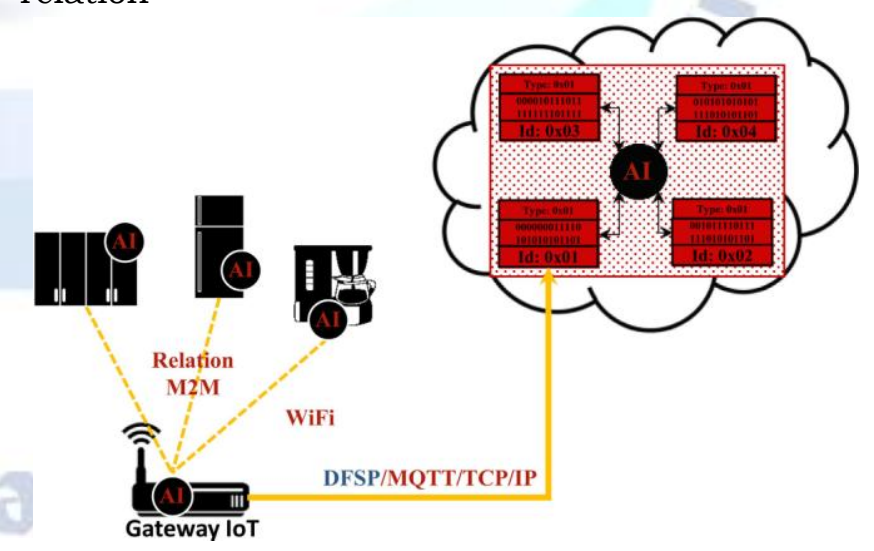

FIGURE 2. Allocation of resources by group of things.

are only made at the gateway level, that is, the central server (MQTT Broker) is not in the cloud.

For this reason, the AI learns about the relation that are commonly established between Things when a group of Things works together. Another 
indispensable resource is to manage access to the internet. The AI will decide whether the packets are forwarded within the same network or outside the network, based on the relation, the resolution of a problem

andaclearreasonfortherequeststobesentoutofthene twork to an IoT platform in the cloud.

IoT Platform + IA: Its function is to maintain network monitoring through the gateway and deliver information only when the gateway requests it.

With the information consulted to Internet through of platform in the cloud [17], the Things on the network can complement the local information and make a better decision.

Example: Using the architecture of Figure 1, all things

are connectedtoeachotherthroughtheGateway(MQTTB roker) + AI. Only the MQTT protocol will be used to establish M2M relations over WiFi connections.. When it detects that there are continuous requests for common activities between the machines, it creates logical work groups. In Figure 2, these three things highlighted in Figure 1 are isolated to explain their relation in more detail. In this case, it has not yet been established who or what resource is needed. This assignment, is the job that the adaptive algorithm of resource allocation controlled by the AI must perform, see Figure 3.

Figure 2 shows a group of Things doing a joint work.

The

M2MrelationthattheAIestablishesdependsonthefun ctions and the service provided by the Things. For example, it could be assumed that something in common between this group of Things is the preparation of Coffee as a service to the User and each Thing can perform a function that helps with the provision of this service. Coffee is a resource that has the kitchen cupboard and milk is a resource that has the refrigerator. The AI activates the algorithm that is responsible for the allocation of resources and the DFSP protocol collects the information.

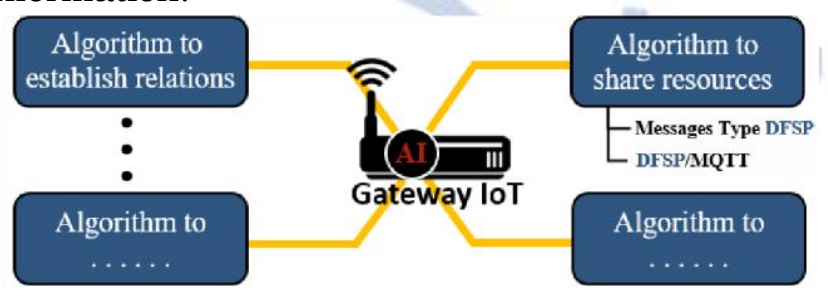

FIGURE 3.IA controlling the algorithms.

In Figure 3, the IoT gateway controls different types of algorithms through the AI and learns from them through the training matrix they provide. Each training matrix is a data file with information about the usage habits and how a machine has been used. This provides information to the system about what kind of operations the user usually performs in front of the machine.

Each petition or service is registered and then analyzed, in order to obtain a statistical history of behavior. After analyzing it with the AI algorithm, things send the resulting matrix to the centralized AI in the gateway. In algorithm 1 , is observe the general steps of the pseudocode of the control algorithm that allows the IA to delegate tasks.

Into the gateway, the AI can handle a number of algorithms and can accommodate as many as necessary and if the capacity of the machine allows it.

The algorithm "share resources" works with the information sent from the Things in the network through the MQTT payload. In the payload, another protocol has been specified that determines the type of information depending on the type of message, called DFSP. The other algorithms provide information and perform tasks in parallel to the other tasks of the network depending on the decisions made by the AI. In this case, it will only control two algorithms, the algorithm to establish relation and share resource.

\section{Algorithm 1 Control Into IoT Gateway}

1.

1) Setaneventlistener(Connectionrequestfrom Clients)

2) Initialize MQTT_Broker

3) Received updates on a subscribed topic

4) Update the information of connected Things

5) Activates the algorithms "establish relation" and "share resources"

6) Supplies initial conditions to the $\mathbf{A I}$

7) Read training matrix of Things

8) Set an event listener (MQTT_Broker)

9) If MQTT_Broker is down then 10)

Disconnect from the Clients

11) end if

12) End.

\section{OPERATION}

IoT technology roadmap: is it a simple list of product/system components, a description of tools, protocols, and application schemes within 
the system or already a blueprint designed to make any product or strategy successful within the IoT ecosystem. In addition to the technical aspect of establishing the IoT roadmap, there are others such as the National IoT Strategic Roadmap and then the corporate roadmap that define IoT on some industrial verticals (Energy, Nanotechnology, Automotive, Cognitive Science ...).

Along with the definition of tech-stack and business-enhancing roadmap processes, it is essential to have clearly documented developmental processes that should in practical terms show the steps that need to be taken into account in the development process.

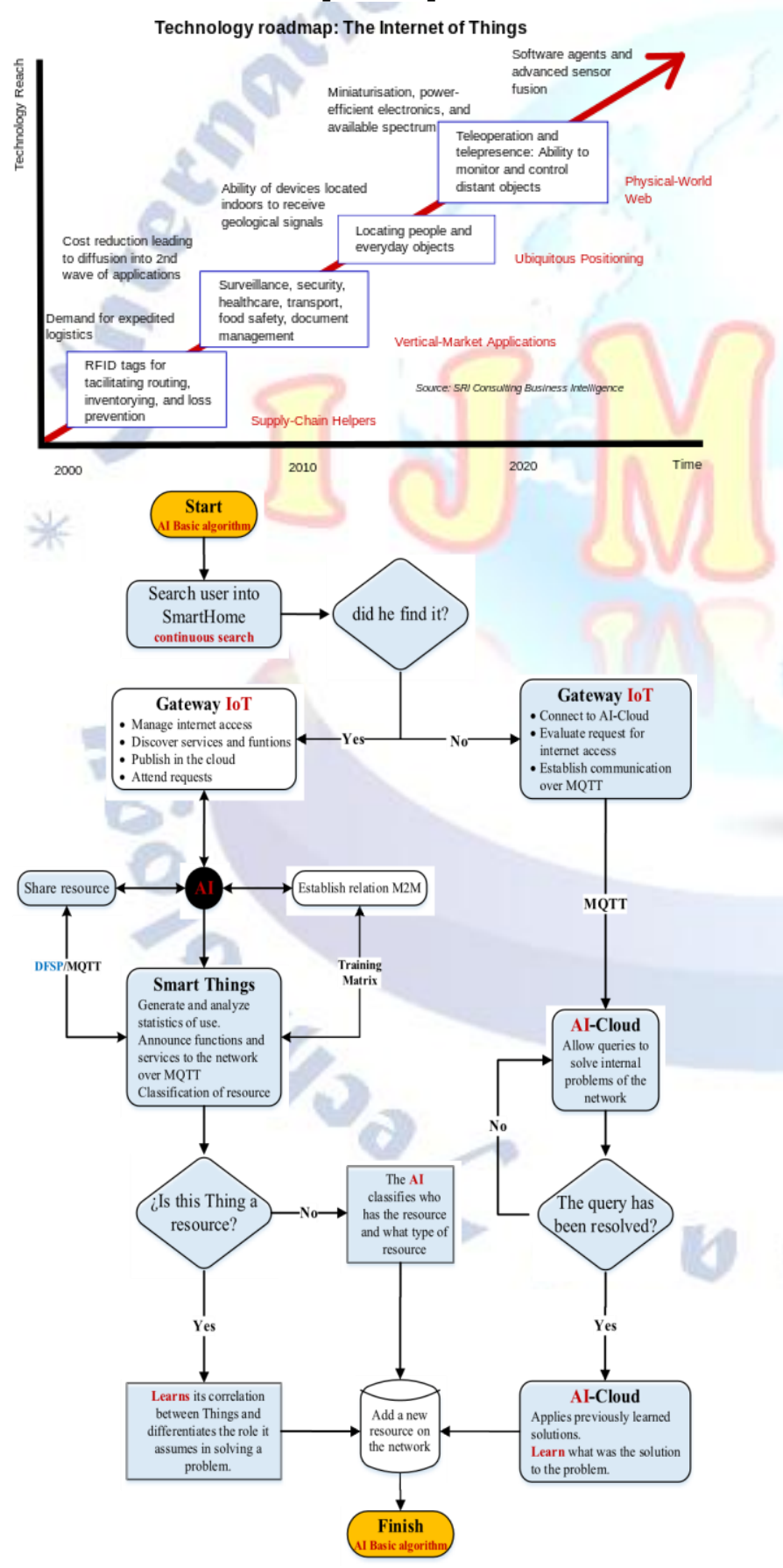

FIGURE 4. Flowchart of the IoT Gateway C AI.
The basic target of an intelligent network is to be able to provide better attention to the user without the need for the latter to control the machines. The idea is that the machines do so autonomously through the AI.

In Figure 4, the AI is observed administering only two algorithms and learning from the user's habits when he is at home. When a problem cannot be resolved internally, the AI decides to search the Internet for possible solutions, learns it and saves it for future problems. The circle that indicates the AI in Figure 4 has the two algorithms that it controls on the sides. Below, the AI analyzes the MQTT payload with the information from the DFSP protocol that comes from Smart Things. Both at the gateway and in smart things $\mathrm{AI}$ are present. However, in the flowchart has represented as only one because both have the same information exchange technique.

The next thing the AI assesses is whether the information that comes through the DFSP is a resource or is a conversation between things. If it is about a conversation that tries to solve a problem, learn it and store it. If it is about a resource then it classifies and stores it. In both cases, the iteration is direct between things, but the information passes through the gateway. Unlike other architectures, the MQTT Broker is present in the Gateway and not in the cloud. This possibly decreases network delays, since for any operation at the local level, it is not necessary to go to a server in the cloud that is far away.

To control internet access, the gateway evaluates whether the requests for things merit it. If the gateway already has, this information previously stored or because you learned it before, then answer the request without having to go to the internet. The cloud platform delivers information requested by the gateway and monitors only what the gateway allows.

\section{E. ALGORITHM TO SHARE RESOURCES}

All the algorithms that the AI manages reside in the IoT Gateway and are based on computational learning techniques (Machine Learning). This algorithm is who allows the Gateway to respond to requests and announcements of resources through the DFSP protocol. The messages of this protocol are encapsulated within the MQTT messages. Its logic is fundamentally based on establishing the role that each Thing plays and the assigning of resources.

The algorithm must know how to differentiate a function or a service from a resource depending on 
the joint work performed by a group of Things. From this, another algorithm controlled by the AI determines the M2M relation of a group of Things. With this information of the groups created by the AI, the algorithm can be adapted dynamically according to the service required by a User.

\section{F. DFSP PROTOCOL}

FunctionandServiceDiscoveryProtocol(DFSP).Thisp rotocol was developed to be introduced within the payload of the PUBLISH messages of the MQTT protocol. That is, everything that is contained in this protocol is a string encoded in UTF.

The main function of this protocol is to discover the functions and services that announce the "Things" when connecting to the network. The algorithm that allows sharing resources in the network takes this information and decides between these two characteristics, if a Thing can be a resource or have a resource. Once the function of this protocol has been defined, the types of messages, their formatting, and the message exchange rules are presented. The header and body of the message of this protocol are simple and like the MQTT header, the size is fixed. Figure 5 shows the protocol format with an example of the ANNUNCEMENT message that transport the FUNTIONS of a thing.

Header: It has a fixed size of 1-Byte with four fields, each of 2-bits (Message Type, Data Type, Flags, RESERVED). In the red box in Figure 5, highlight the protocol header and below is the body. The format of the messages is shown in 8-bit linear datagrams per $\mathrm{n}$-Bytes (8-bits $\mathrm{x}$ n-Bytes) one above the other.

\begin{tabular}{|c|c|c|c|c|c|c|c|c|}
\hline $\begin{array}{l}\text { Messag } \\
0 \text { - ANN } \\
1 \text {-DISCC }\end{array}$ & $\begin{array}{l}\text { e Type } \\
\text { UNCEN } \\
\text { OVER }\end{array}$ & & & $\begin{array}{l}\text { pe } \\
\text { IIONS } \\
\text { ICES }\end{array}$ & $\begin{array}{l}\text { Flags } \\
0-N \\
1-M\end{array}$ & $\begin{array}{l}\text { ages } s \\
\text { ger }\end{array}$ & & \\
\hline bit & 7 & 6 & 5 & 4 & 3 & 2 & 1 & 0 \\
\hline & Mess & Type & & ype & & & RES & VED \\
\hline Byte 1 & 0 & 0 & 0 & 0 & 0 & 0 & $x$ & $\mathrm{x}$ \\
\hline & & & ( & H" (0 & 48) & & & \\
\hline Byte 2 & 0 & 1 & 0 & 0 & 1 & 0 & 0 & 0 \\
\hline & & & & E" $(0$ & 48) & & & \\
\hline Byte 3 & 0 & 1 & 0 & 0 & 0 & 1 & 0 & 1 \\
\hline & & & & L" $(0$ & 4C) & & & \\
\hline Byte 2 & 0 & 1 & 0 & 0 & 1 & 1 & 0 & 0 \\
\hline & & & & L" $(0$ & 4C) & & & \\
\hline Byte 2 & 0 & 1 & 0 & 0 & 1 & 1 & 0 & 0 \\
\hline & & & & $\mathrm{O}^{\prime \prime}(0$ & $4 F)$ & & & \\
\hline Byte 2 & 0 & 1 & 0 & 0 & 1 & 1 & 1 & 1 \\
\hline Byte 2 & & & & '," (0x & 2C) & & & \\
\hline & 0 & 0 & 1 & 0 & 1 & 1 & 0 & 0 \\
\hline$\vdots$ & $\vdots$ & $\vdots$ & $\vdots$ & $\vdots$ & $\vdots$ & 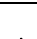 & $\vdots$ & \\
\hline$\vdots$ & $\vdots$ & $\vdots$ & $\vdots$ & $\vdots$ & $\vdots$ & $\vdots$ & $\vdots$ & $\vdots$ \\
\hline
\end{tabular}

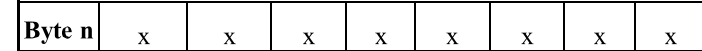

FIGURE 5. DFSP protocol format.

Body: The body carries the information of the type of message that has been defined in the header. This information is a string encoded in UTF, starting from the second Byte up to 250-MBytes. This information is PERFORMANCE TEST

In this section the performance of the test is provide. The tests of this proposal are made with the target of demonstrating that the algorithm can make decisions to assign resources depending on the information collected by the DFSP protocol.

\section{A. IMPLEMENTATION OF THE DEVICES}

The development of this proposal involves the use of singleboard computers (SBC) such as the Raspberry Pi 3 Model B+ (RPi3) [19]. Each actor in this proposal, it has implemented with an RPi3, except for the cloud platform. It has tested on two different platforms for IoT: ThingsBoard [20] and ThingSpeak [21].

The idea is that each thing is controlled by an RPi3 including the IoT Gateway, under the Android Things operating system [22]. Using the Java programming language and the Android Studio development environment [23].

\section{B. TESTBED}

The technical implementation of this proposal is possible through the implementation of the RPi3, three of it emulating three Smart Things and one emulating the IoT Gateway. Another way to verify the operation of this proposal can be done through a simulation. Several simulators were studied, including Cooja [24], NS-2 [25] (Network Simulator 2), GNS3 [26] and Cisco Packet Tracer [27]. Most of the simulators are oriented to IoT sensor networks (Motes, mostly 8 and 16 bit Microcontroller based). Some of these simulators do not support the use of the RPi3 because it falls into the SBC category and cannot run a full Operating System (OS). Sometimes also, the OS of the simulators are not compatible with OS of RPi3. Currently, there is an RPi3 simulator [28], [29] that can emulate the operating system (OS-RPi3), but cannot simulate it in a network. 


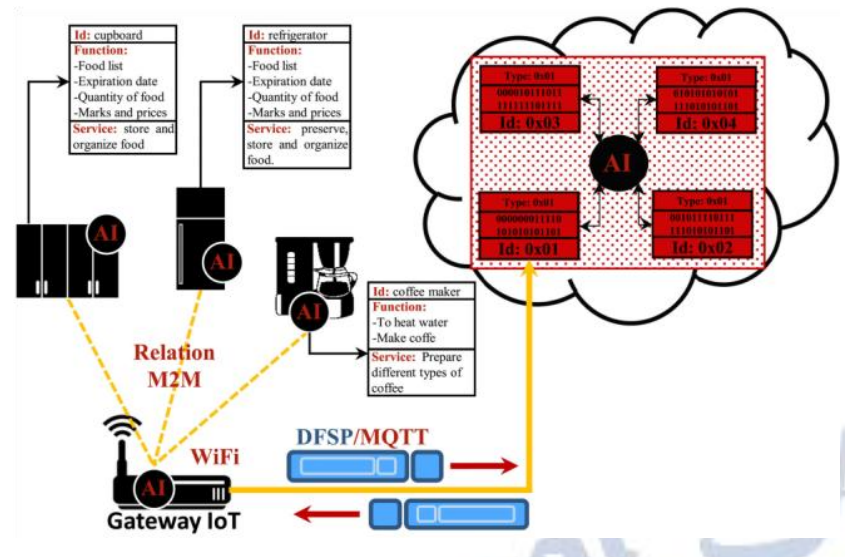

FIGURE 7. Operation of the proposal.

Cisco Packet Tracer simulator was the best option in this case, although it has limitations when emulating the speed of processing of the packets and the time of decision in the AI algorithm. This simulator allows illustrating the behavior of the $\mathrm{RPi} 3$ in a network environment and seeing the operation of the MQTT protocol. It also allows you to modify the preinstalled sample programs and adjust them to the design of this proposal.

The modified program was the MQTT Client and MQTT Broker protocol in Python code into each RPi3. An algorithm was added to both to calculate the length of each of the 16 messages of the MQTTV3.1.1 protocol [30], called Remaining Length (RL). This algorithm can also be understood mathematically knowing that the MQTT packet or message format consists of a Header (always present) + Variable Header (not always present) + Payload (not always present) [31]. The above depends on the type of message. The following figure illustrates the MQTT packet format.

The MQTT packet size (PS) is calculated according to the type of message through the following equation (1).

$$
\mathrm{PS}=[\mathrm{C}+\mathrm{XL}]+\mathrm{RL}, \text { Bytes }
$$

The length of the Control field (C) will always be 1 Byte and if the value to be saved in the Packet length field (PL) is less than or equal to 127 , then the header will have a fixed value of 2 Bytes. However, if the value calculated by the RL is greater than 127, the length of the PL field changes, modifying the total size of the package (PS).

The packet length field (PL) saves the size of the calculated packet and can occupy a length of between 1 and 4 bytes. To know how many bytes it is used and how is to save the size in this field, it used the following equation (2).

$$
X L=\sum_{\mathrm{n}=0}^{3} X_{\mathrm{n}^{*}} 128^{\mathrm{n}}
$$

$\mathrm{XL}$ is the calculation in Bytes needed to represent the number that corresponds to the value of RL, and that will be transported within the PL field located in the header. The representation of this number in Hex can occupy a space of the range between 1-Bytes to 4-Bytes. Since the length of this

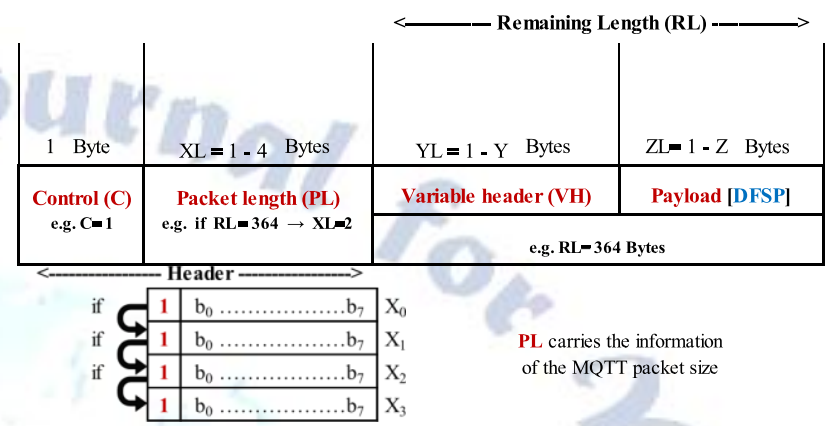

FIGURE 8. MQTT packet format. TABLE 1. Equations of the algorithm RL.

As can be seen in Figure 11, the length of the packets shows that the DFSP protocol has been introduced in the payload of MQTT compared to what was seen in Figure 10.

The length of the TCP packets given during the test is shown in Figure 11. The maximum values are peaks of 209 bytes, while the minimum value has been 81 bytes, which belongs to an UNSUBSCRIBE message. The average number of bytes transferred has been 116 Bytes.

Figure 12 shows the relation between numbers of packets is transmitted per seconds along the tests. The captured time is the real time of duration each packet sent in the network, which is measured in milliseconds (ms). The maximum time transmission of packets was at $0.1 \mathrm{~ms}$, with 5 packets. The rest of the packets remain constant after the connection is established through the TCP socket.

In this time interval, PUBLISH/SUBSCRIBE messages are exchanged. The average time of the TCP packets transporting them is $0.070 \mathrm{~ms}$.

In Figure 13, it is can see the data transfer rate (DTR) in Bytes per seconds between each Smart Thing and the IoT

Gateway. In the simulation, the AI makes a boot of the smart

FIGURE 13. Data transmission rate. 


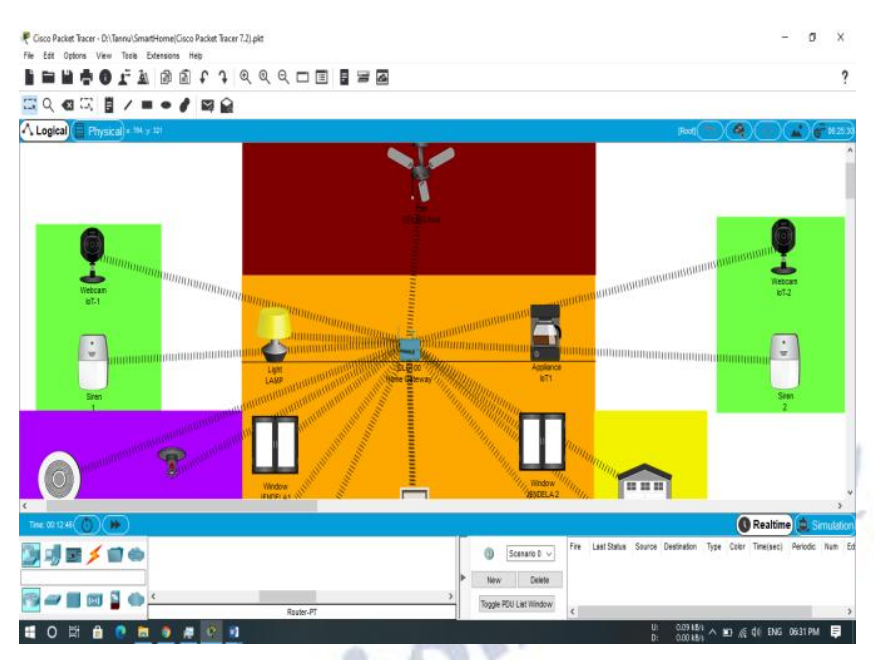

FIGURE 14. Implementation .

things automatically and start to send the messages of MQTT protocol to the Gateway.

In Figure 13, the transmission of a message different from the MQTT protocol is observed in a sequential test process performed by the AI. This sequence begins with the CONNECT message and ends with the DISCONNECT message.Thebitrateofeachtransmissionfromthecus tomers is on average between $1000 \mathrm{Bps}$ and 2100 Bps. In the disconnection segment, a higher speed is observed, on average, between $2100 \mathrm{Bps}$ and 3600 Bps.

In the simulation seen in Figure 14 has added to each Thing (fridge, cupboard and coffee maker) a training matrix based on statistics of usage and user preferences.

These data are assumed through a text file, in order to observe the operation of the system and thus be able to capture the packages evidencing the transport of information autonomously to other machines.

The simulation also allows us to observe the functioning of Things by controlling other things with basic functions such as on/off (on, red color), selection of type coffee preparation and the ingredients available for its preparation.

In a real implementation within a smart home, everything will be smart [35], [36]. Each of the things in this proposal, including the gateway, is built with an RPi3 and programmed with AI. As shown in Figure 12, it also connects to the cloud where there is also a platform with AI. As in the simulation, this proposal is expected to use other types of connections such as Bluetooth 5.0, WiFi and Ethernet. The idea is that protocols M2M, in this case, MQTT can be transported throughout the network, regardless of the type of the underlying connection.

\section{CONCLUSION}

In this paper, a smart home system was designed and implemented using Cisco Packet Tacer using Node MCU microcontroller enabkes with Wi-Fi as a method of monitoring and controlling home appliances i,e user remotely access the system from anywhere around the world.The system is capable of automating the operation of the appliances by analyzing the regular usage patter of the appliances by the user.This not just saves a lot of human effort, but also helps in conserving energy.Also, it can help the differently abled and the elderly in performing basic tasks at home such as switching on/off the light,fan, and so on without havingto dependon others.

\section{FUTURE WORK}

IoT involves enhancing network to proficiently collect and analyze the data from various sensors and actuators then send the data to the mobile phone of a personal computers over a wireless connection, building IoT has progressed essentially in the last couple of years since it has created a new era in the world of information's and communication technologies. Security is an important issue now days, as the possibilities of intrusion are increasing day by day. Safety from intrusion, theft, fire and leakage gas are most important requirements of home security system for the peoples.

\section{ACKNOWLEDGMENT}

I thank my Mentor Ass. Prof. Mrs Shikha Gupta for analyzing the data and for advising on all aspects related to this paper, along with other concepts too. Later for validating the experimental result and reviewed the paper.

\section{REFERENCES}

[1] "Home Automation and Security System with Node MCU using Internet of Things",K. Lova Raju, V. Chandrani, SK. Shahina Begum, M. Pravallika Devi, IEEE 2019.

[2] "Smart-Home Automation using IoT-based Sensing and Monitoring Platform",Majid Al-Kuwari, Abdulrhman Ramadan, Yousef Ismael, Laith Al-Sughair, Adel Gastli, Mohieddine Benammar ,IEEE 2019.

[3] "Smart Energy Efficient Home Automation System Using IoT", Satyendra K. Vishwakarma, Prashant Upadhyaya, Babita Kumari, Arun Kumar Mishra, IEEE 2019.

[4] "A Review on Home Automation using Voice Via Bluetooth Through Raspberry PI3”,Jayant Dorve, Manish K. Samarth, 
Swapnil R. Jais, Md. Danish S. Sheikh, Pawan Kumar, Hanuman Korde, IJRESM 2019.

[5] Chattoraj, Subhankar. "Smart Home Automation based on different sensors and Arduino as the mastercontroller." International Journal of Scientific andResearch Publications5.10 (2015): 1-4. "Advanced Irrigation System using Arduino and Raspberry Pi as Centralized Server",N. Kishore, K. Ashok kumar, IRJET 2019.

[6] "Smart Gardening Automation using IoT With BLYNK App" , Mitul Sheth, Pinal Rupani ,IEEE 2019.

[7] "Home Automation System Based on IOT using Cellular Devices", Ravi Wankhade, Shashank Karhade, Pratik Mohite, Kanchan Dhole, Akash Ganvir, Bharti Khedkar, Sharayu Sangekar, IJSRST 2019.

[8] "IoT Based Smart Home Garden Watering System Using Raspberry Pi 3", Sandhya.B.R, Pallavi.M, Chandrashekar.M, IJIRSET 2018.

[9] S. Haller S. Karnouskos and C. Schroth "The Internet of Things in an Enterprise Context " in Future Internet-FIS International Journal of Engineering Science Invention Research \& Development; Vol. IV, Issue VII, JANUARY 2018 Lecture Notes in Computer Science Vol. 5468 2009pp 14-28. "Cost Effective Autonomous Plant Watering Robot", Mahendra Vucha, K Jyothi, Kiran Kumari, R Karthik, IJRTE 2019.

[10] Soliman, Moataz, et al. "Smart home: Integrating internet of things with web services and cloud computing." Cloud Computing Technology and Science (CloudCom), 2013 IEEE 5th International Conference on. Vol. 2. IEEE, 2013.

[11] Jie, Yin, et al. "Smart home system based on iot technologies." Computational and Information Sciences(ICCIS), 2013 Fifth International Conference on. IEEE, 2013

[5]http://www.packettracernetwork.com/internet-ofth ings/pt7-iot-devicesconfiguration.htm

[12] S. Raja Gopal, P. Saleem Akram , S. Sriram, T. Pavan Koushik, V. Mohana Krishna, -Design and Analysis of Heterogeneous Hybrid topology for VLAN configuration-, International Journal of Emerging Trends in Engineering Research, Vol 7, No 11, PP 487 - 491, 2019.

[13] J. Rocher, M. Taha, L. Parra, and J. Lloret, "IoT sensor to detect fraudulent use of dyed fuels in smart cities," in Proc. 5th Int. Conf. Internet Things, Syst., Manage. Secur.

[14] P. Srikanth Reddy, P. Saleem Akram, M. Adarsh Sharma, P. Aditya Sai Ram, R. Pruthvi Raj, -Study and Analysis of Routing Protocols-, International Journal of Emerging Trends in Engineering Research, Vol 7, No 11, PP 434 440, 2019.

[15] Rambabu K., Venkatram N. Traffic flow features as metrics (TFFM): Detection of application layer level DDOS attack scope of IOT traffic flows -, 2018, International Journal of Engineering and Technology(UAE) ,Vol: 7 ,Issue: 1.1 ,pp: 554 to:: 559 ,DOI: ,ISSN: $2227524 X$. 